\title{
Indirekte Auswirkungen von Naturgefahren auf den Tourismus - Das Beispiel des Lawinenwinters 1999 in der Schweiz
}

\author{
Christian J. Nöthiger, Davos, Hans Elsasser, Zürich, \\ Michael Bründl, Walter J. Ammann, Davos
}

\section{Ausgangslage}

Katastrophale Naturereignisse können nicht alleine zu Todesopfern und Sachbeschädigungen, sondern auch zu Mindereinnahmen für die Wirtschaft führen. Dies wurde in der Schweiz bisher auf wissenschaftlicher und politischer Ebene kaum thematisiert. Während der Lawinenereignisse im Februar 1999 wurde das Thema aber durch die Massenmedien aufgegriffen, insbesondere in Bezug auf die Tourismusbranche in den Alpen. Es zeigte sich dabei nicht nur, dass kaum Strategien existieren, um allfällige Mindereinnahmen zu reduzieren. Es wurde auch offensichtlich, dass alleine schon zum möglichen Ausmass dieser Mindereinnahmen praktisch nichts bekannt ist. Dies gilt allerdings nicht nur für die Alpen und für Lawinen, sondern auch für katastrophale Naturereignisse im Allgemeinen. Das ist umso problematischer, weil im Rahmen des prognostizierten globalen Klimawandels immer auch die Zunahme katastrophaler Naturereignisse postuliert wird, selbst wenn im konkreten Fall der Schadenlawinenhäufigkeit bislang kein Zusammenhang zum Klimawandel nachweisbar ist (vgl. Schneebeli, Laternser \& AmmanN 1997).

Trotzdem bot sich gerade der Lawinenwinter 1999 für das Eidg. Institut für Schnee- und Lawinenforschung SLF als Ausgangspunkt an, um diese sogenannten «indirekten» Auswirkungen von Naturgefahren - speziell für den Tourismus - näher zu untersuchen. Fernziel ist es, bei zukünftigen Ereignissen in der Schweiz die indirekten Kosten möglichst schnell auf einfache Art abschätzen zu können sowie Massnahmen zur Senkung dieser Kosten vorzuschlagen. In diesem Artikel können erste Ergebnisse der bisherigen Untersuchungen präsentiert werden. Zunächst wird aber kurz allgemein auf den Lawinenwinter und auf Probleme im Zusammenhang mit indirekten Auswirkungen eingegangen.

\section{Der Lawinenwinter 1999: Wetterlage, Lawinenabgänge, Todesopfer}

Der Winter 1998/99 wird vielen Bewohnern der Alpen als sehr aussergewöhnlich in Erinnerung bleiben. Zwi- schen dem 27. Januar und dem 25. Februar 1999 folgten kurz hintereinander drei intensive Niederschlagsperioden, die von stürmischen Nordwestwinden begleitet waren. Am Alpennordhang fielen innerhalb von 30 Tagen verbreitet über $5 \mathrm{~m}$ Neuschnee. Das ist mehr als sonst in einem ganzen Winter. Diese aussergewöhnlichen Schneefälle hatten eine grossräumige, sehr intensive Lawinenaktivität zur Folge. Alleine in der Schweiz wurden im Winter 1998/99 über 1000 Schadenlawinen gezählt. Die grösste Häufung von Lawinenabgängen fiel jeweils mit den drei Starkschneefallperioden um den 29. Januar, den 9. Februar und den 22. Februar zusammen. Die starken Nordwestwinde, welche die über fast einen Monat anhaltenden Schneefälle verursacht hatten, führten auch zu umfangreichen Triebschneeansammlungen, was die Situation zusätzlich verschärfte. Zudem war die Stabilität der ganzen Schneedecke nur mässig. Durch diese Effekte in Kombination mit einem markanten Temperaturanstieg kam es zwischen dem 20. und 23. Februar zur grössten Lawinenaktivität des Winters. Erstmals seit Einführung der einheitlichen europäischen Lawinengefahrenskala 1993 kamen in der Schweiz im Februar 1999 während längerer Zeit die beiden höchsten Gefahrenstufen zur Anwendung. Für sechs Tage wurde die höchste Stufe, d.h. "sehr grosse» Lawinengefahr, prognostiziert (vgl. SLF 2000: 27-97).

Durch Lawinenabgänge kamen im Februar 1999 im gesamten Alpenraum auf Verkehrswegen und in Gebäuden 71 Menschen ums Leben, davon 12 in Frankreich, 17 in der Schweiz und 41 in Österreich (IKAR 1999). Am stärksten betroffen waren die Gemeinden Galtür, Österreich (31 Tote), Evolène, Schweiz (12 Tote) und Chamonix, Frankreich (12 Tote). Überdies starben bei Freizeitaktivitäten im Laufe des gesamten Winterhalbjahres 1998/99 in den Alpen weitere 74 Menschen durch Lawinen.

\section{Direkte und indirekte Kosten des Lawinenwinters}

Das Eidgenössische Institut für Schnee- und Lawinenforschung SLF verfasste im Auftrag des Bundesamtes für Umwelt, Wald und Landschaft BUWAL/ Eidg. Forstdirektion, Bern eine Ereignisanalyse des Lawinenwinters 1999 in der Schweiz (SLF 2000). Darin wird auch auf die durch die Lawinenabgänge verursachten Schadenkosten eingegangen. 


\begin{tabular}{lr} 
Schadensbereich & $\begin{array}{r}\text { Geschätzter } \\
\text { Schaden }\end{array}$ \\
\hline Gebäude & 194 Mio. SFr. \\
Fahrhabe (Hausrat) & 58 Mio. SFr. \\
Strassen & 63 Mio. SFr. \\
Schiene & 11 Mio. SFr. \\
Bergbahnen & 17 Mio. SFr. \\
Schutzbauten & 9 Mio. SFr. \\
Elektrizitätsnetz & 27 Mio. SFr. \\
Wald & 46 Mio. SFr. \\
Landwirtschaftsflächen & 14 Mio. SFr. \\
\hline Total & $\mathbf{4 3 9}$ Mio. SFr. \\
\hline
\end{tabular}

Tab. 1: Übersicht über die direkten Lawinen- und Schneedruckschäden des Lawinenwinters 1999 in der Schweiz

The cost of direct damages caused by avalanches and snow pressure during the avalanche winter of 1999 in Switzerland

Aperçu des coûts des dommages directs provoqués en Suisse par les avalanches et la pression de la neige en hiver 1999

Bei den Auswirkungen von katastrophalen Naturereignissen muss grundsätzlich zwischen direkten und indirekten Schäden bzw. Kosten unterschieden werden. Unter direkten Schäden versteht man Kosten, die durch direkte Einwirkung des Ereignisses an Gebäuden, Infrastrukturanlagen, Wald- und Landwirtschaftsflächen verursacht werden. Indirekte Schäden sind die Folgekosten eines katastrophalen Naturereignisses, die über die Räumungs- und Wiederinstandsetzungskosten von direkten Schäden hinausgehen. Darunter fallen insbesondere Mindereinnahmen für die Wirtschaft (Produktionsausfälle oder -erschwernisse, Verluste an Marktanteilen etc.). In den Alpen ist in erster Linie die Tourismusbranche von diesen Mindereinnahmen betroffen, weshalb sich dieser Artikel auch darauf konzentriert.

Direkte Kosten sind grösstenteils durch Versicherungen oder die öffentliche Hand abgedeckt und damit auch erfassbar. Im Falle des Lawinenwinters 1999 ergab sich für die gesamte Schweiz eine Schadensumme von etwa 439 Mio. SFr. (vgl. Tabelle 1). Alleine 194 Mio. SFr. davon entfallen auf Gebäudeschäden. Der überwiegende Teil dieser Schäden entstand an
Wohn- und Landwirtschaftsgebäuden. Gastgewerblich genutzte Gebäude waren dagegen nur sehr selten und meist in geringem Ausmass betroffen (vgl. Abbildung 1). Die bekannte Schadensumme an Hotelbetrieben und Gaststätten beträgt lediglich etwa 3 Mio. SFr. Auch die 17 Mio. SFr. Schadenkosten bei Bergbahnund Skiliftanlagen betreffen speziell die Tourismusbranche. Allerdings war hier mit $3 \%$ ebenfalls nur ein sehr geringer Teil aller Anlagen der Schweiz betroffen. Dies bedeutet, dass Tourismusbetriebe hauptsächlich von indirekten Kosten betroffen waren, während sich die direkten Schäden in Grenzen hielten.

Bei den indirekten Kosten eines katastrophalen Naturereignisses handelt es sich grösstenteils um aufgrund des Ereignisses nicht erzielte Einnahmen. Während die Kosten für Reparaturen und Räumungen spätestens nach Abschluss der Arbeiten bekannt sind, bleiben Vermutungen über entgangene Einnahmen letztlich immer hypothetisch. Es können einzig Vergleiche mit Vorjahresperioden angestellt und so Mindereinnahmen abgeschätzt werden. Weil dies aufwändig und mit zahlreichen Unsicherheiten behaftet ist, wurde bei bisherigen katastrophalen Naturereignissen in der Schweiz jeweils weitgehend darauf verzichtet (vgl. z.B. Krisenstab Brig 1994: 18).

\section{Durchgeführte Erhebungen zur Grundlagengewinnung}

Der einzige Bereich, aus dem sich zu wirtschaftlichen Folgen des Lawinenwinters 1999 Schlüsse aus der amtlichen Statistik ziehen lassen, sind die Logiernächte in Hotelbetrieben. Diese werden in der Schweiz monatlich veröffentlicht und sind bereits etwa zwei Monate nach der Erhebungsperiode greifbar (vgl. BFS 1993-2001). Mangels Alternativen waren diese Daten die Grundlage der Schätzungen zu den wirtschaftlichen Auswirkungen des Lawinenwinters. Unklar ist dabei aber unter anderem, inwiefern sich aus einem Logiernächterückgang in der Hotellerie Rückschlüsse auf andere Zweige des Tourismusgewerbes ziehen lassen. Es mussten also zusätzliche Angaben erhoben werden.

Auch wenn die indirekten Kosten des Lawinenwinters nirgends statistisch erfasst sind, war doch davon auszugehen, dass die betroffenen Unternehmen im Einzelfall durchaus in der Lage wären, die ihnen entstandenen Mindereinnahmen ungefähr abzuschätzen. Als Erhebungsmethode für die indirekten Schäden des Lawinenwinters drängten sich also Befragungen auf. Hauptproblem dabei war die grosse Zahl der Betroffenen.

Um den Aufwand zu beschränken, wurden zwei 


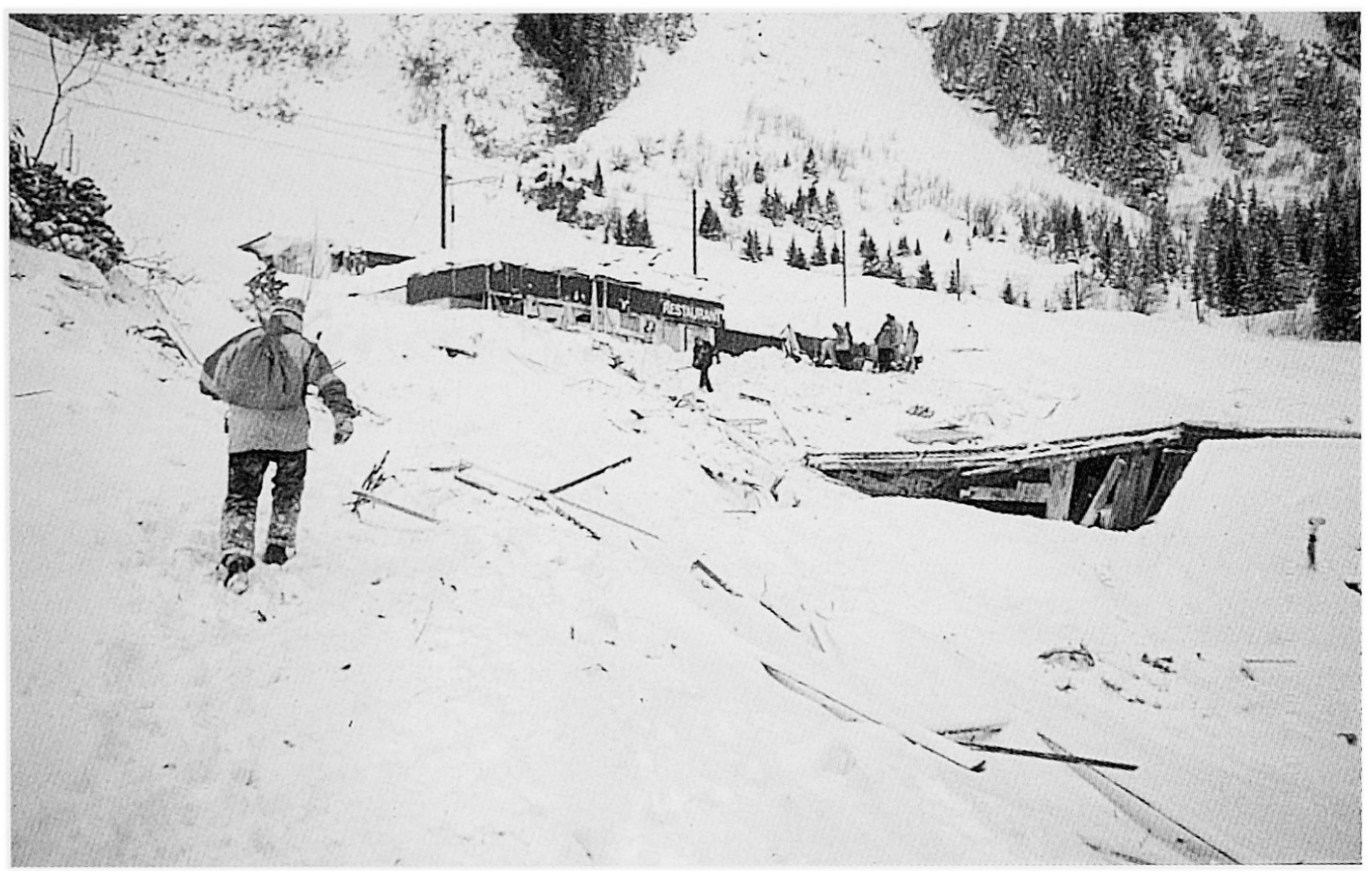

Abb. 1: Das am 8. Februar 1999 durch einen Lawinenabgang zerstörte Restaurant «Oberland» in Wengen (Kanton Bern), in welchem das Wirtepaar den Tod fand, stellt einen tragischen Ausnahmefall dar: Sonst wurden in der Schweiz im Februar 1999 nur selten Gastwirtschaftsbetriebe von Lawinen beschädigt.

The destruction of the "Oberland" restaurant in Wengen (Canton of Berne) by an avalanche on 8 February 1999, and the death of the couple who owned it, is a tragic exception: Only a few hotel or restaurant buildings in Switzerland were damaged by avalanches during February 1999.

La destruction du restaurant "Oberland» à Wengen (Canton de Berne) par une avalanche le 8 février 1999, lors de laquelle les propriétaires ont trouvé la mort, représente une exception tragique. En Suisse, les bâtiments hôteliers n'ont été en effet que rarement endommagés par les avalanches de février 1999.

Foto: Kantonspolizei BeRn, 8.2.1999 (Abdruck mit freundlicher Genehmigung)

Gemeinden ausgewählt, in denen jeweils Unternehmensbefragungen durchgeführt wurden. Die Gemeinden waren Davos im Kanton Graubünden, einer der logiernächtestärksten Ferienorte der Schweiz sowie, als Beispiel für eine kleinere Gemeinde, Elm im Kanton Glarus, welche vor allem von Tagestouristen aus dem Raum rund um den Zürichsee frequentiert wird. Davos war im Februar 1999 während dreier Tage wegen Lawinengefahr von der Aussenwelt abgeschnitten. In Elm wurde die einzige Zufahrtsstrasse von einer Grosslawine verschüttet und war erst nach zehn Tagen wieder passierbar (vgl. Abbildung 2).

In die Unternehmensbefragung in Davos wurden Hotels, Gaststätten, Bergbahnen, Schneesportschulen, der Detailhandel sowie Industrie- und Gewerbebetriebe einbezogen (vgl. Nöthiger \& AmmanN 2001). In Elm konnten zudem auch Vermieter von
Gruppenunterkünften und Ferienhäusern befragt werden (vgl. NöTHiger 2000). Insgesamt wurden dabei 473 strukturierte Fragebogen (vgl. STENGER 1998: 41) versandt, wovon $58 \%$ zurückgesandt wurden. Um nicht nur die Anbieterseite zu erfassen, wurden in beiden Gemeinden zusätzlich Gästebefragungen durchgeführt. Erfasst wurden sowohl Personen, die im Februar 1999 in Davos oder Elm eingeschlossen waren, als auch solche, welche die Ereignisse des Lawinenwinters von zu Hause aus mitverfolgten. Aus diesen Befragungen liegen 130 ausgefüllte Fragebogen vor.

Die Umfragen in den beiden Gemeinden ergaben ein ziemlich gutes Bild der lokalen Folgen des Lawinenwinters 1999 für die verschiedenen Wirtschaftszweige. Was aber fehlte, war ein Überblick über die Gesamtschweiz, der im Bereich des Tourismus die Hotellogiernächtestatistik hätte ergänzen können. Um 


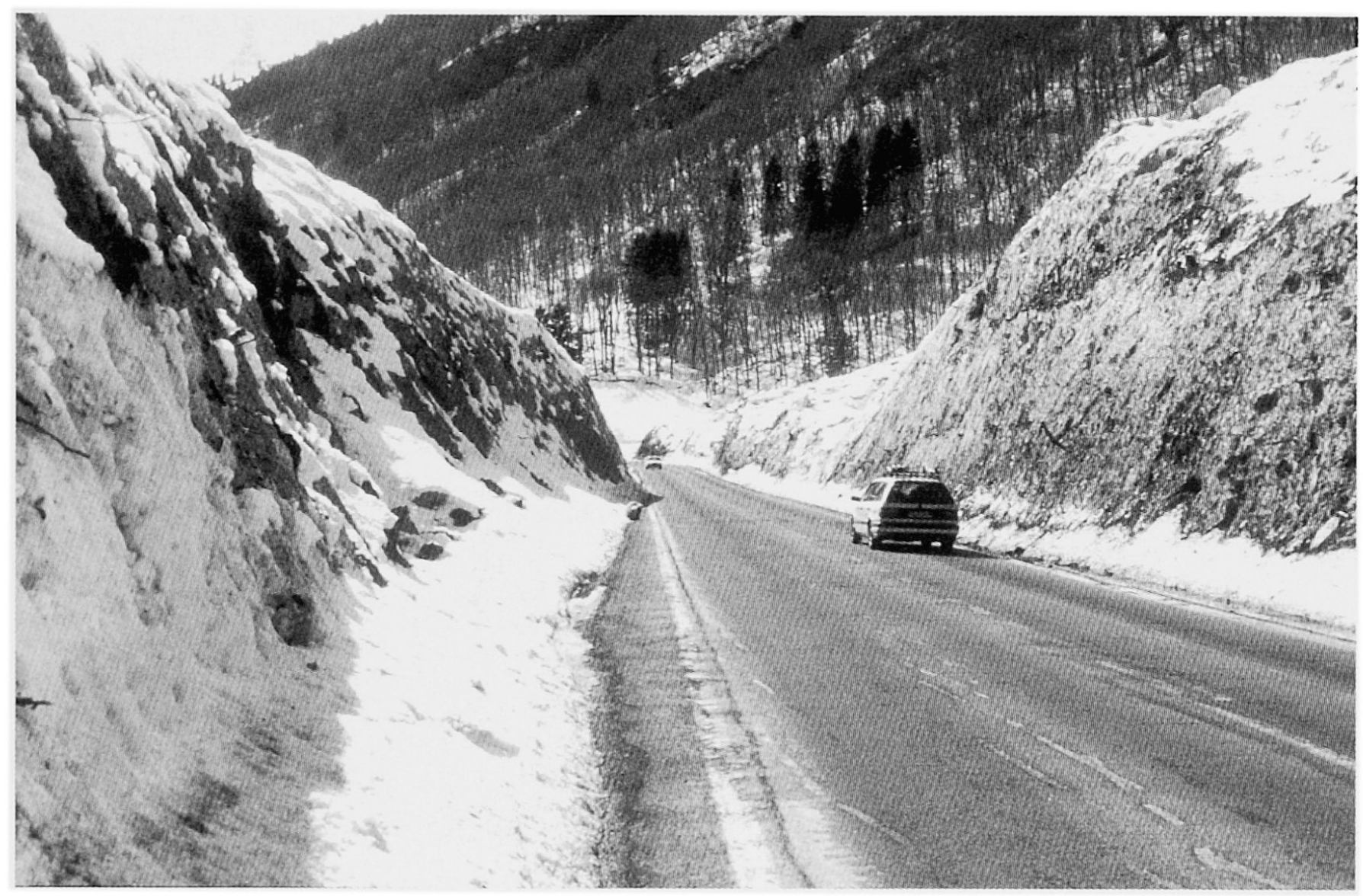

Abb. 2: Die frisch geräumte Kantonsstrasse zwischen Matt und Elm (Kanton Glarus), welche durch die Meissenbodenlawine im Februar 1999 während zehn Tagen hintereinander blockiert gewesen war.

The newly cleared cantonal road between the villages of Matt and Elm (Canton of Glarus), which was blocked by the «Meissenboden» avalanche for ten successive days in February 1999.

La route cantonale entre les villages de Matt et Elm (Canton de Glaris) fraîchement déblayée après son blocage par l'avalanche de "Meissenboden» pendant dix jours successifs.

Foto: CH. NöTHIGER, 10.3.1999

diesen Überblick zu gewinnen, wurde eine Umfrage bei Bergbahnunternehmen lanciert (vgl. NöTHIGER, BRÜNDL \& AMMANN 2001). Diese eignen sich dafür vor allem, weil sie für das Winterhalbjahr mit Sicherheit das wichtigste touristische Angebot darstellen. Die Umfrage fand erst im Sommer 2000 statt. Dies erlaubte es, auch allfällig längerfristige Folgen des Lawinenwinters $\mathrm{zu}$ erfassen. Insgesamt wurden die 117 grössten Bergbahn- und Skiliftgesellschaften der deutsch- und französischsprachigen Schweiz befragt. Die Rücklaufquote war mit $79 \%$ ausserordentlich gut.

\section{Erste Erkenntnisse zu den indirekten Kosten des Lawinenwinters 1999}

\subsection{Wie entstehen indirekte Kosten?}

Bei einem direkten Lawinenschaden stellt sich die Frage nach der genauen Schadensursache nicht. Bei indirekten Kosten ist diese dagegen schwerer zu eruieren. Am einfachsten ist die Sachlage, wenn ein Betrieb derart beschädigt wird, dass dort nicht mehr gearbeitet werden kann. Die indirekten Kosten entsprechen dann den Einnahmen, die in der Zeit bis zur Wiederinstandsetzung der Schäden nicht erzielt werden konnten. Wie oben gezeigt wurde, war aber gerade im Gastgewerbe nur eine sehr geringe Zahl von Betrieben von direkten Schäden betroffen.

Der grösste Teil der indirekten Kosten hatte also andere Ursachen. Ein wichtiger Faktor waren sicher die zahlreichen Sperrungen oder Blockierungen von Verkehrsachsen. Da die Zahl der Zufahrtswege zu Orten im Berggebiet im Allgemeinen begrenzt ist, wurden in der Schweiz im Februar 1999 über 150 Ansiedlungen eine Zeitlang von der Aussenwelt abgeschnitten (vgl. Abbildung 3). Darunter waren auch 42 Fremdenverkehrsorte, welche bis maximal 14 Tage 


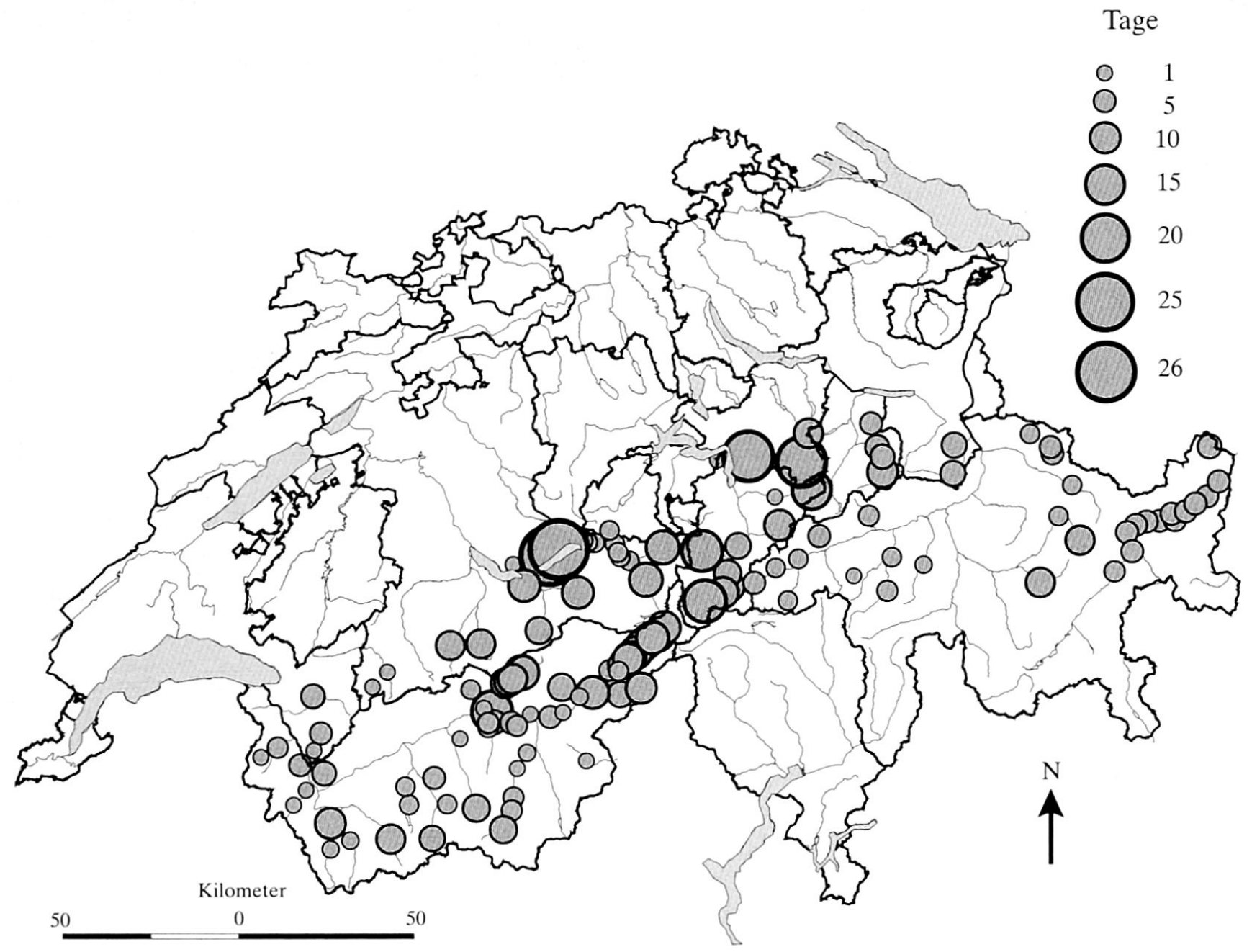

Abb. 3: Gemeinden und Weiler, die im Februar 1999 auf der Strasse eine Zeitlang nicht mehr erreichbar waren. Die Kreisgrösse ist proportional zur längsten ununterbrochenen Sperrung sämtlicher Zufahrtsstrassen in Tagen. Villages no longer accessible by road during a period of time in February 1999. The size of the circles is proportionate to the longest continuous closure of all access roads in days.

Les villages qui n'étaient plus accessibles par la route pendant un certain temps en février 1999. La taille des cercles est proportionnelle à la plus longue durée ininterrompue du blocage de toutes les routes d'accès (en nombre de journées).

Quelle: SLF (Eidgenössisches Institut für Schnee- und Lawinenforschung) 2000: 225

ohne Unterbruch isoliert waren. Hier entstanden indirekte Kosten, weil der Tagestourismus während dieser Zeit natürlich komplett ausfiel. Bei längeren Sperrungen kam es vielerorts aber auch zur Evakuierung von übernachtenden Gästen auf dem Luftweg (vgl. Abbildung 4). Diese Massnahme wurde besonders dort durchgeführt, wo sich die Sperrungen über Wochenenden hinwegzogen, über die klassischen An- und Abreisetage in Wintersportgebieten also. Da nur in seltenen Fällen auch Gäste eingeflogen wurden, wirkte sich eine Sperrung der Zufahrten über das Wochenende besonders negativ auf die Logiernächtezahlen aus.

Indirekte Kosten entstanden aber nicht nur in den
Fremdenverkehrsorten, die während des Lawinenwinters von der Umwelt abgeschnitten waren. Von den in der SLF-Umfrage erfassten Bergbahn- und Skiliftunternehmen machten nur $26 \%$ eine Sperrung der Zufahrten für den Rückgang ihrer Passagierzahlen im Februar 1999 verantwortlich (vgl. Abbildung 5). Für $48 \%$ fiel dagegen ins Gewicht, dass verschiedene ihrer Anlagen wegen Lawinengefahr still standen. Man kann davon ausgehen, dass im Februar 1999 etwa 45\% der Bergbahn- und Skiliftanlagen im schweizerischen Alpenraum während durchschnittlich sieben Tagen ausser Betrieb waren (NÖTHIGER, BRÜNDL \& AMMANN 2001: 27f.). Dadurch fielen trotz geöffneter Zufahrtswege Tagesgäste aus, was nicht nur für die Bergbahn- 


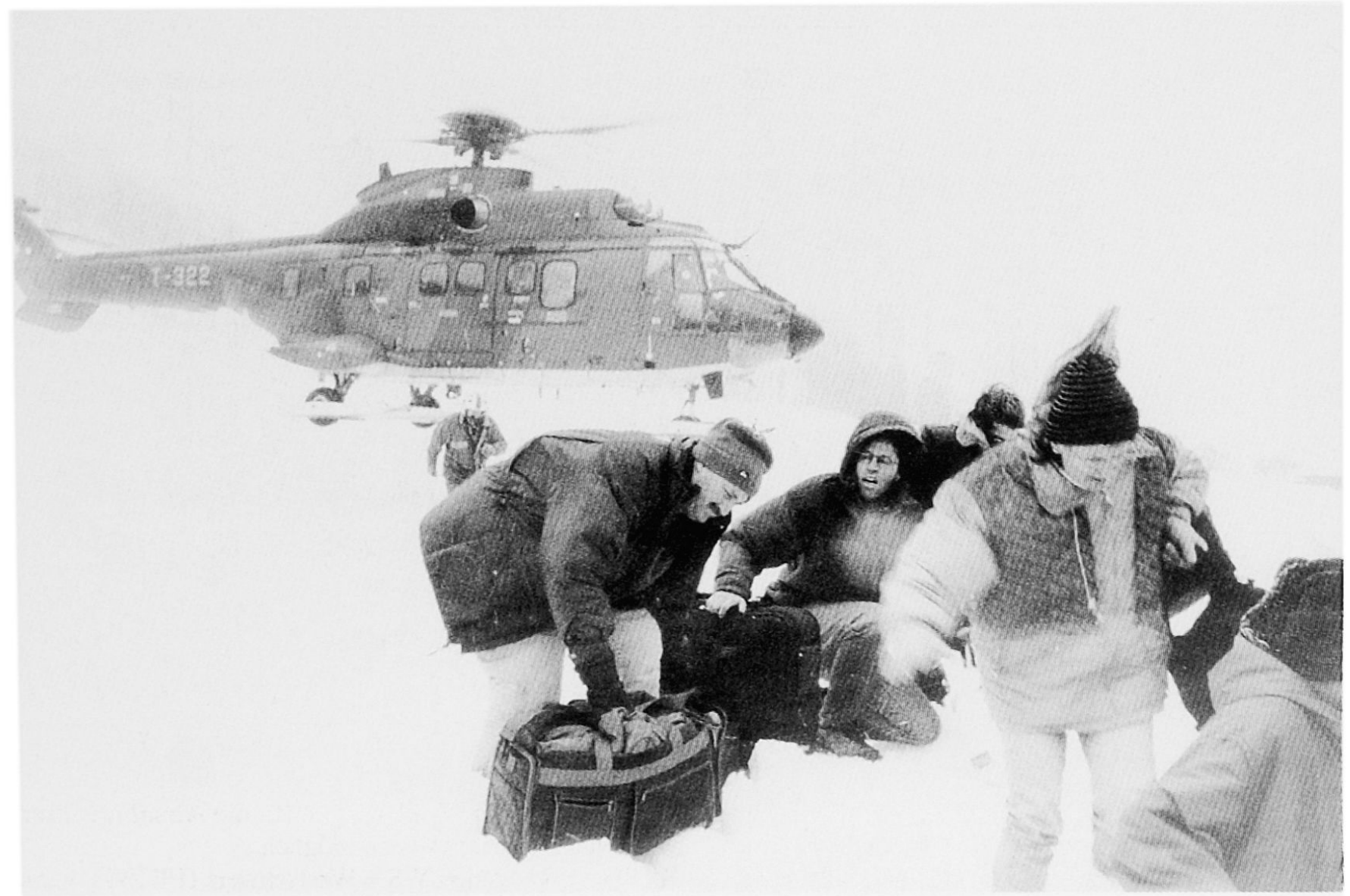

Abb. 4: Evakuierung von Touristen mit einem Helikopter der Schweizer Luftwaffe aus dem Sernftal (Kanton Glarus), nachdem im Februar 1999 sämtliche Strassenverbindungen durch Lawinenabgänge blockiert worden waren.

Tourists being evacuated from the "Sernf» valley (Canton of Glarus) by a Swiss Air Force helicopter after all access roads had been blocked by avalanches in February 1999.

Evacuation, en février 1999, des touristes de la vallée du Sernf (Canton de Glaris) par hélicoptère des forces aériennes suisses après le blocage de toutes les routes d'accès par les avalanches.

Foto: E. von ARB, 20.2.1999 (Abdruck mit freundlicher Genehmigung, Redaktion «Die Südostschweiz»)

unternehmen, sondern auch für die übrige Tourismusbranche in den betroffenen Orten negative Folgen hatte.

Doch auch geschlossene Anlagen können noch nicht hinreichend erklären, warum im Februar 1999 im schweizerischen Alpenraum $88 \%$ der Bergbahn- und Skiliftunternehmen Mindereinnahmen und $60 \%$ der Fremdenverkehrsorte einen Logiernächterückgang zu verzeichnen hatten (vgl. dazu Abschnitt 5.4). Dafür lassen sich aber zwei weitere Ursachen anführen, deren Einfluss allerdings weder unterscheidbar noch quantifizierbar ist: Einerseits lud das Wetter während der intensiven Niederschlagsperioden, die im Februar 1999 letztlich zu den Rekordschneemengen geführt hatten, kaum zum Wintersport ein (vgl. Abbildung 6). Eine nachhaltige Wetterbesserung trat erst in der letzten Februarwoche ein, welche aber zugleich die Woche mit der grössten Lawinenaktivität war. Dies dürfte wahrscheinlich dazu geführt haben, dass sich auch am letzten Februarwochenende, als das Wetter gesamtschweizerisch betrachtet noch am besten war, nicht allzu viele Wintersportler auf die Pisten wagten. Es erstaunt deshalb nicht, dass $60 \%$ der befragten Bergbahn- und Skiliftunternehmen das schlechte Wetter im Februar für ihre Mindereinnahmen verantwortlich machten.

Sogar $63 \%$ gaben an, sie sähen in übertriebenen Medienberichten zur Lawinengefahr eine weitere ausschlaggebende Ursache für ihre Mindereinnahmen. Diese Medienkritik ist auch in der übrigen Tourismusbranche weit verbreitet. $89 \%$ der befragten Betriebe in der Gemeinde Davos betrachteten die Medienberichterstattung zum Lawinenwinter als $\mathrm{zu}$ negativ und machten sie für einen Imageschaden verantwortlich, 


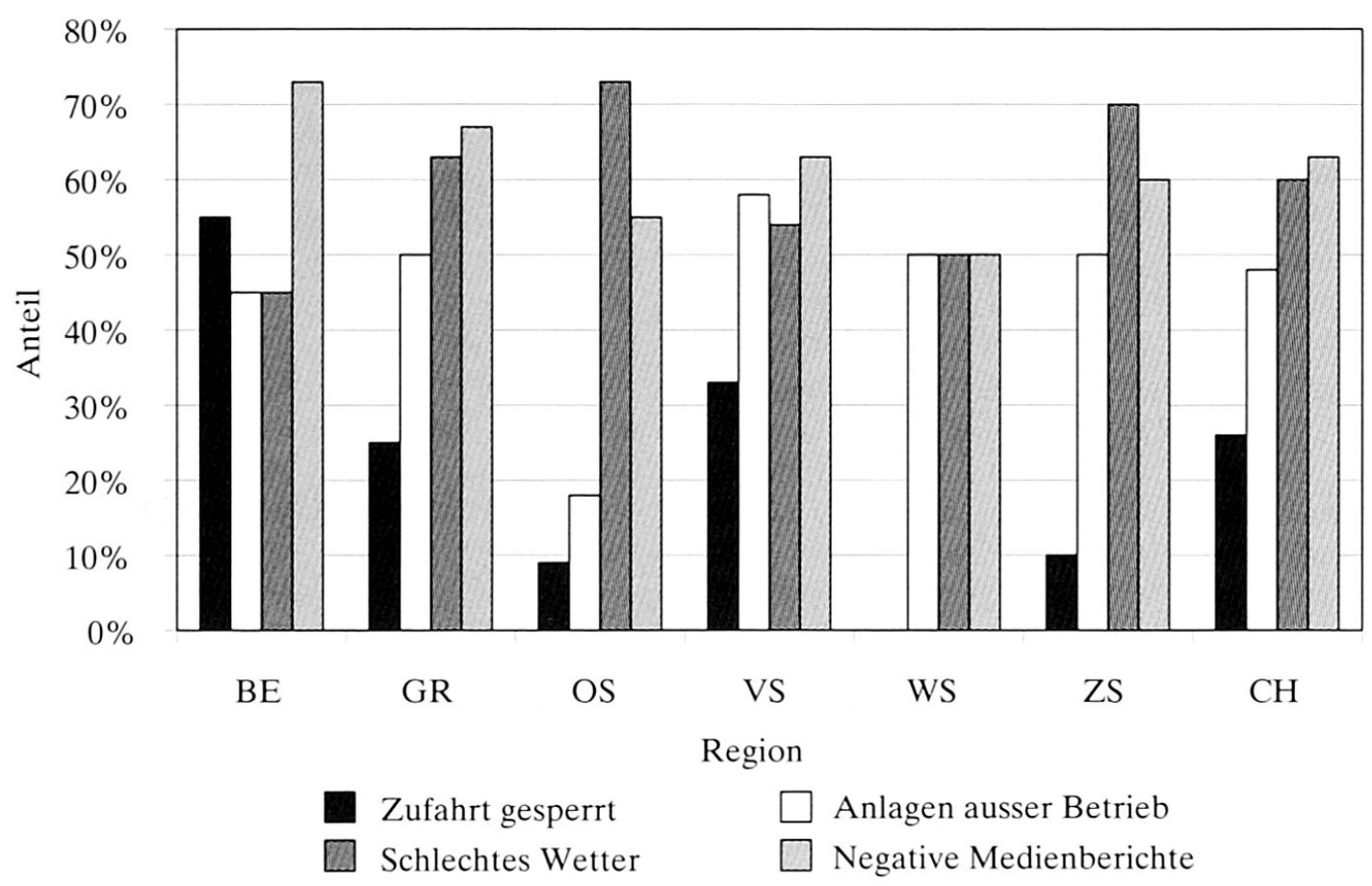

Abb. 5: Von den Schweizer Bergbahn- und Skiliftunternehmen angeführte Erklärungen für die Abnahme ihrer Passagierzahlen im Februar 1999 (in \% aller Betriebe). Mehrere Antworten waren möglich.

$\mathrm{BE}=$ Bern, GR = Graubünden, OS = Ostschweiz (AI, GL, SG), VS = Wallis, WS = Westschweiz (FR, NE, VD), $\mathrm{ZS}=$ Zentralschweiz (LU, NW, OW, SZ, UR), $\mathrm{CH}=$ Gesamtschweiz (ohne Tessin)

Explanations for the decrease of the number of their passengers during February 1999 quoted by the Swiss cable car and ski lift companies (in percentages of the total number of companies). More than one reason was possible. Explications données par les sociétés de remontées mécaniques concernant la diminution du nombre de leurs passagers en février 1999 (en pourcentage de toutes les entreprises). Plusieurs réponses étaient possibles.

Quelle: NÖTHIGER, BrüNDL \& AMMANN 2001:28.

während nur $11 \%$ sie für fair und korrekt hielten (NöTHiger \& Ammann 2001:9). Hauptkritikpunkt war, dass die Lage zu sehr verallgemeinert wurde. So sei der Eindruck entstanden, der gesamte Alpenraum sei eine Gefahrenzone. Zudem hätten die Medien, als die Lawinengefahr wieder abgenommen hatte, zu wenig darüber berichtet. Viele potentielle Gäste hätten so gar nicht gewusst, dass die Wintersportverhältnisse wieder gut wären.

Bei der Beurteilung dieser Vorwürfe gilt es zu bedenken, dass die Medien in jedem Fall nur über einen besonders interessanten Ausschnitt aus der Realität berichten können. Die Anforderung, ein Geschehen wie den Lawinenwinter 1999 in seiner ganzen Komplexität «umfassend» darzustellen, vermag die Nachrichtenberichterstattung auch nicht annähernd zu erfüllen. Das Urteil darüber, ob «korrekt» berichtet wurde, ist also letztlich subjektiv. Es ist allerdings kaum auszuschliessen, dass Medienberichte einen gewissen Einfluss auf potentielle Gäste von Wintersportgebieten haben; nur schon, weil sie im Normalfall schlicht die einzige Infor- mationsquelle zur Lage vor Ort sind. Wie gross dieser Einfluss ist, und ob die Berichte die Situation im Falle eines Naturereignisses noch schlimmer machen, als sie ohnehin schon ist, kann allerdings mit wissenschaftlichen Mitteln kaum beantwortet werden.

5.2 Wer bezahlte die direkten und indirekten Kosten? Am Beispiel der Gemeinde Elm konnte genau untersucht werden, wer bis zu welchem Grad in die Deckung der indirekten Kosten des Lawinenwinters involviert war. Da dies zugleich auch für die direkten Schadenkosten möglich war, können interessante Unterschiede aufgezeigt werden. Man kann davon ausgehen, dass sie für Gemeinden im schweizerischen Alpenraum typisch sind.

Tabelle 2 stellt die Verteilung der Kosten auf die nationale, kantonale und kommunale Ebene dar. Neben den Beiträgen des Bundes werden auch die Privatversicherungen und Hilfswerke zur nationalen Ebene gezählt, weil diese im Normalfall in der ganzen Schweiz operieren. 


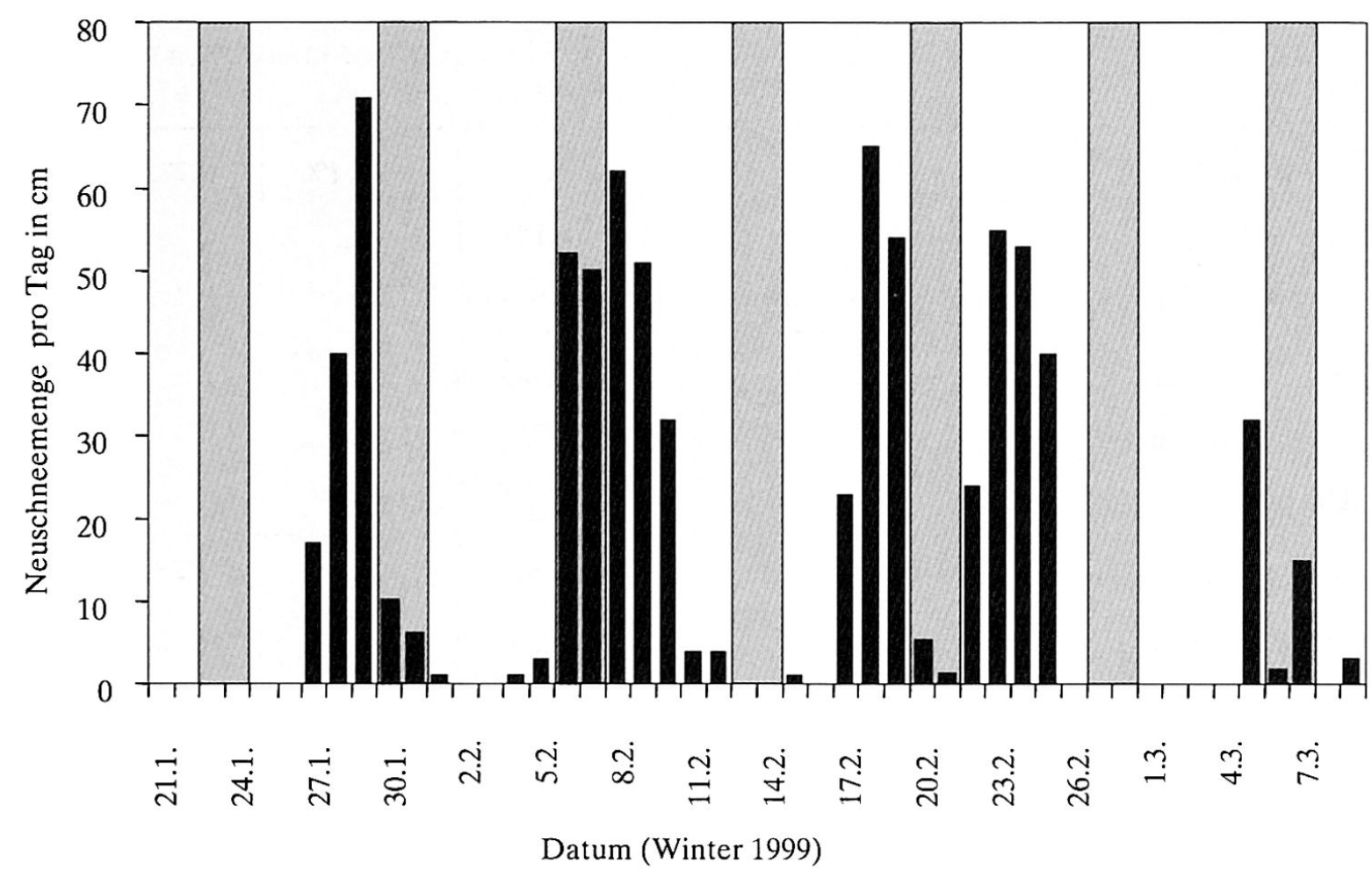

Abb. 6: Zeitliche Entwicklung der Schneefälle im Februar 1999 in Braunwald (Kanton Glarus) auf 2470 m ü. M. Graue Balken = Wochenenden.

Temporal development of snowfall during February 1999 in Braunwald (Canton of Glarus) at 2470 meters above sea level. The grey columns indicate weekends.

Développement temporel des chutes de neige à Braunwald (Canton de Glaris), à $2470 \mathrm{~m}$ d'altitude. Les colonnes grises marquent les fins de semaine.

Quelle: EIDGENÖSSISCHES INSTITUT Für SCHNEE- UND LAWINENFORSCHUNG SLF, Davos

In der Tabelle wird deutlich erkennbar, dass die öffentliche Hand für den grössten Teil der direkten Kosten aufkam. Auf kantonaler Ebene waren vor allem die öffentlichen Versicherungen von Bedeutung, in erster Linie die kantonale Gebäudeversicherung. Etwa ein Fünftel aller direkten Kosten war von privaten Versicherungen gedeckt. Den von direkten Schäden betroffenen Betrieben und Haushalten verblieben so am Schluss nur die Selbstbehalte der Versicherungen. Als Einzige nicht durch Versicherungen oder andere Mittel gedeckt, waren gewisse Schäden an Privatstrassen.

Ganz anders sieht das Bild bei den indirekten Kosten des Lawinenwinters aus: Die öffentliche Hand war nur über die Arbeitslosenkasse in die Kostendeckung involviert. Betriebe, in denen mangels Kundschaft nicht gearbeitet werden konnte, bekamen aus dieser Kasse einen Anteil an den weiterlaufenden Lohnkosten vergütet. In einem Fall wurde ein Tourismusbetrieb beschädigt, der eine Betriebsunterbrechungsversicherung abgeschlossen hatte. Eine solche Versicherung entschädigt Fixkosten und entstandene Mindereinnah- men in der Zeit, während der dort aufgrund eines Schadens nicht gearbeitet werden kann. Liegt kein Gebäudeschaden vor, sondern muss der Betrieb z.B. wegen blockierten Verkehrsachsen eingestellt werden, zahlt eine Betriebsunterbrechungsversicherung im Normalfall aber nicht.

Der ganze Rest der indirekten Kosten - immerhin 93\% der Gesamtsumme - ging deshalb zu Lasten der Betroffenen, in erster Linie Betriebe des Tourismussektors. Es ist mit Sicherheit ein typisches Merkmal indirekter Kosten von Naturereignissen, dass die Betroffenen in diesem Bereich nicht auf die gesamtgesellschaftliche Solidarität zählen können. Besonders die Ausfälle in Hotels, Restaurants und Läden betrafen die einzelnen Betriebsinhaber teilweise recht stark. Es scheint aber in der Schweiz ein politischer Konsens darüber zu bestehen, dass es sich dabei um unternehmerisches Risiko handelt. Dies ganz im Gegensatz zur Landwirtschaft übrigens, bei der nicht nur in Elm auch die indirekten Kosten des Lawinenwinters durch die öffentliche Hand und private Hilfswerke praktisch vollständig abgedeckt waren. 


\begin{tabular}{|l|r|r|r|}
\hline & \multicolumn{1}{|c|}{$\begin{array}{c}\text { Direkte } \\
\text { Kosten }\end{array}$} & $\begin{array}{c}\text { Indirekte } \\
\text { Kosten }\end{array}$ & $\begin{array}{c}\text { Gesamt- } \\
\text { kosten }\end{array}$ \\
\hline Nationale Ebene & $\mathbf{3 6 . 7} \%$ & $\mathbf{0 . 4} \%$ & $\mathbf{2 2 . 3} \%$ \\
Davon Bund & $14.1 \%$ & - & $8.5 \%$ \\
Davon private Versicherungen/Hilfswerke u.ä. & $22.6 \%$ & $0.4 \%$ & $13.8 \%$ \\
\hline Kantonale Ebene & $\mathbf{4 9 . 0} \%$ & $\mathbf{6 . 6} \%$ & $\mathbf{3 2 . 3} \%$ \\
Davon Kanton & $9.3 \%$ & - & $5.7 \%$ \\
Davon öffentliche Versicherungen & $39.7 \%$ & $6.6 \%$ & $26.6 \%$ \\
\hline Kommunale Ebene & $\mathbf{1 4 . 3} \%$ & $\mathbf{9 3 . 0} \%$ & $\mathbf{4 5 . 4 \%}$ \\
Davon Gemeinde & $8.2 \%$ & - & $5.0 \%$ \\
Davon Betriebe, Haushalte u.a. & $6.1 \%$ & $93.0 \%$ & $40.4 \%$ \\
\hline
\end{tabular}

Tab. 2: Prozentuale Verteilung der Kosten des Lawinenwinters 1999 in Elm (Kanton Glarus) auf die nationale, kantonale und kommunale Ebene.

Proportional distribution of costs resulting from the avalanche winter of 1999 in Elm (Canton of Glarus) on the national, cantonal and local level.

Distribution proportionnelle des coûts des dommages provoqués par les avalanches de l'hiver 1999 à Elm (Canton de Glaris) aux échelles nationale, cantonale et communale.

Quelle: NÖTHIGER 2000: 38

\subsection{Gibt es auch positive indirekte Auswirkungen?}

Ein Grossteil der direkten Schadenkosten des Lawinenwinters entsprechen den Ausgaben für Wiederinstandsetzungsarbeiten, welche letztlich v.a. Baufirmen oder Handwerkern zugute kommen. Man kann deshalb davon ausgehen, dass der Industrie- und Gewerbesektor als Ganzes eher vom Lawinenwinter profitiert hat. Dies bestätigten auch die SLF-Umfragen in Elm und Davos (NöTHiger \& AMmanN 2001: 8 und NÖTHIGER 2000:33f.): Neben Reparaturarbeiten wirkte sich dort auch die Räumung der zusätzlichen Schneemengen finanziell positiv aus. Die grosse Mehrheit der Industrie- und Gewerbebetriebe wurde vom Lawinenwinter trotz gesperrter Zufahrtswege kaum tangiert, weil sie flexibel auf die Situation reagieren konnten. Negativ betroffen waren einzig Betriebe, die direkt vom Tourismus abhängen (z.B. Tankstellen, Skireparaturservice u.ä.). Es stellt sich an dieser Stelle deshalb vor allem die Frage, ob auch für das Tourismusgewerbe allenfalls positive Effekte des Lawinenwinters zu verzeichnen waren.

Es wäre beispielsweise denkbar, dass viele Schneesportler im Februar 1999 auf lawinensichere Gebiete ausgewichen sind und dass jene davon profitiert hätten. Die Umfrage unter Bergbahn- und Skiliftunterneh- men zeigte, dass dieser Effekt tatsächlich gespielt hat, allerdings nur in geringem Masse. So sagten immerhin $10 \%$ der Unternehmen aus, dass sich ihre Passagierzahlen bzw. Einnahmen auf dem Vorjahresniveau gehalten hätten (NöTHIGER, BRÜNDL \& AMMANN 2001: 28). Angesichts der Wettersituation im Februar 1999 ist dies wohl bereits als Erfolg zu werten. Die betreffenden Unternehmen befinden sich grösstenteils in den Voralpen und im Jura (vgl. Abbildung 7). In den Westschweizer Voralpen und im Jura hatten sogar in jedem vierten Betrieb die Passagierzahlen und Einnahmen zugenommen. Es wurden jedoch keine Angaben gemacht, wie gross die Zunahme der Einnahmen ausgefallen war. Es kann aber ausgeschlossen werden, dass die Mehreinnahmen bei vereinzelten Betrieben die Mindereinnahmen bei der grossen Mehrheit der Bergbahn- und Skiliftunternehmen auch nur annähernd aufgewogen hätten.

Auch sogenannte «Sensationstouristen» müssen im Zusammenhang mit allfällig positiven wirtschaftlichen Auswirkungen des Lawinenwinters berücksichtigt werden. Darunter werden Schaulustige verstanden, welche nach einem katastrophalen Naturereignis als Tagestouristen die Orte des Geschehens besuchen. Ob diese Sensationstouristen bei der betroffenen einhei- 


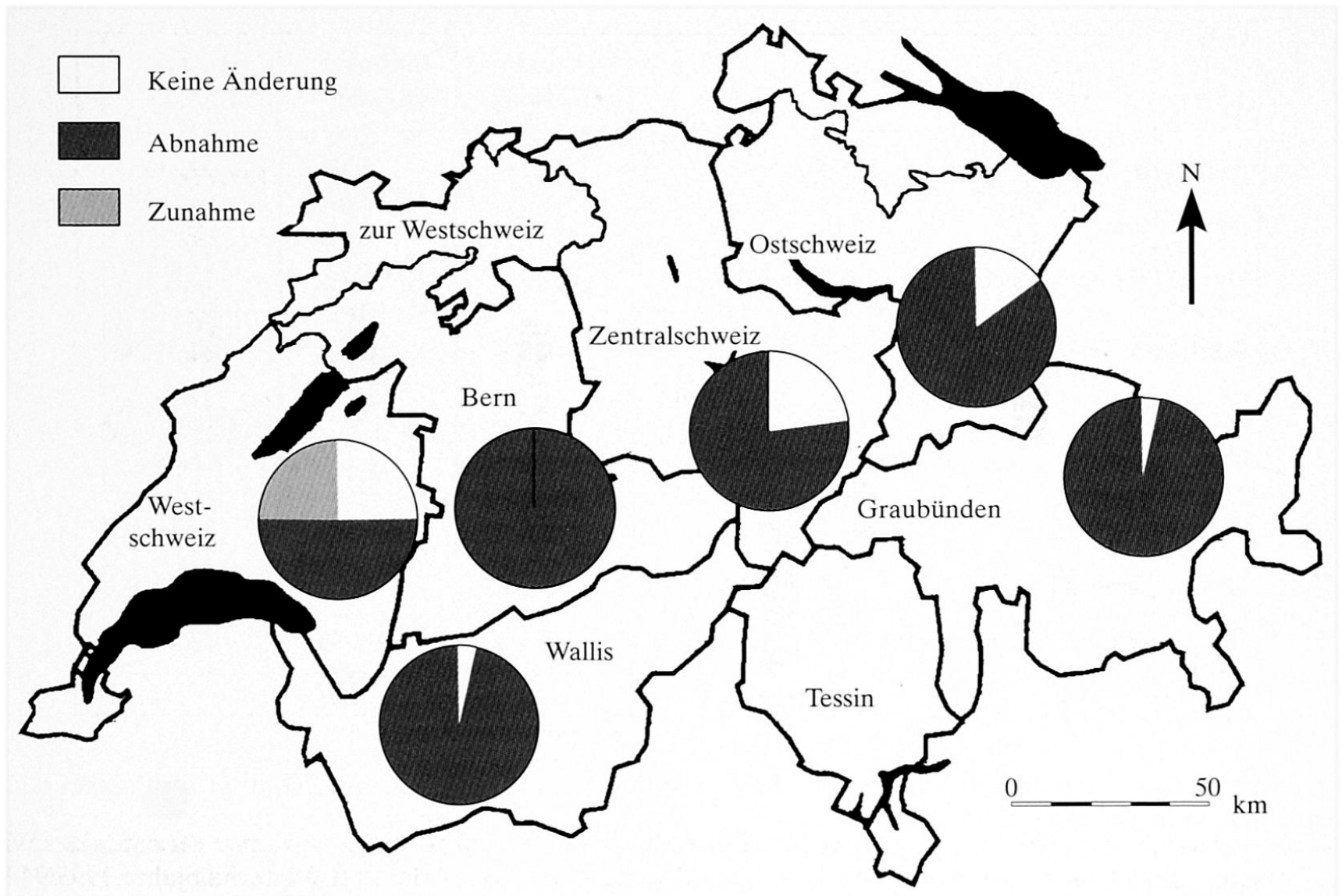

Abb. 7: Einfluss des Lawinenwinters auf die Passagierzahlen bzw. Einnahmen der Schweizer Bergbahn- und Skiliftunternehmen im Februar 1999 verglichen mit Februar 1998 in den einzelnen Regionen der Schweiz. Angaben in \% aller Betriebe der jeweiligen Region.

Influence of the avalanches of February 1999 on the number of passengers resp. the earnings of the cable car and ski lift companies in different regions of Switzerland compared to February 1998 (in percentages of the respective total number of companies in each particular region)

Impact des avalanches de février 1999 sur le nombre des passagers ainsi que sur le chiffre d'affaires des sociétés de remontées mécaniques par rapport au mois de février de 1998, dans les différentes régions de la Suisse. En pourcentage du nombre total respectif des entreprises dans la région concernée.

Quelle: NÖTHIGER, BRÜNDL \& AmMANn 2001: 28

mischen Bevölkerung eher auf Sympathie stossen oder nicht, soll hier nicht Gegenstand der Diskussion sein. $\mathrm{Zu}$ ihrer wirtschaftlichen Bedeutung liegen dagegen Erkenntnisse vor.

Zunächst einmal ist das Auftreten von Sensationstouristen davon abhängig, was es überhaupt zu sehen gibt. Die Umfragen zeigten nämlich, dass in der Gemeinde Elm recht viele Sensationstouristen beobachtet wurden, während dies in Davos überhaupt nicht der Fall war (Nöthiger \& AMmANN 2001: 6 und NöTHIGER 2000: 30). Ursache dürften sicher die spektakulären Lawinenkegel in unmittelbarer Nähe des Siedlungsgebietes gewesen sein, die zwar in Elm vorhanden waren, in Davos dagegen nicht. In Elm zeigte sich auch, wer von den Sensationstouristen profitieren konnte. Die Sensationstouristen reisten überwiegend im eigenen Auto an, hielten sich im Dorfkern auf und besuchten vor ihrer Abreise allenfalls noch eine Gaststätte. Profitieren konnten deshalb nur die Gaststätten im Dorfkern und die Tankstelle, beispielsweise aber nicht die Bergbahnen, der Detailhandel oder der Busbetrieb. Die befragten Betriebe konnten die zahlenmässige oder finanzielle Bedeutung der Sensationstouristen nicht quantifizieren. Es ist aber doch mit ziemlicher Sicherheit davon auszugehen, dass auch die in bestimmten Orten aufgetretenen Sensationstouristen die Mindereinnahmen des Lawinenwinters nicht wirklich wettmachen konnten.

\subsection{In welchem Zeitraum treten negative indirekte Auswirkungen auf?}

Während die Entstehung direkter Schäden eines katastrophalen Naturereignisses zeitlich unmittelbar an 


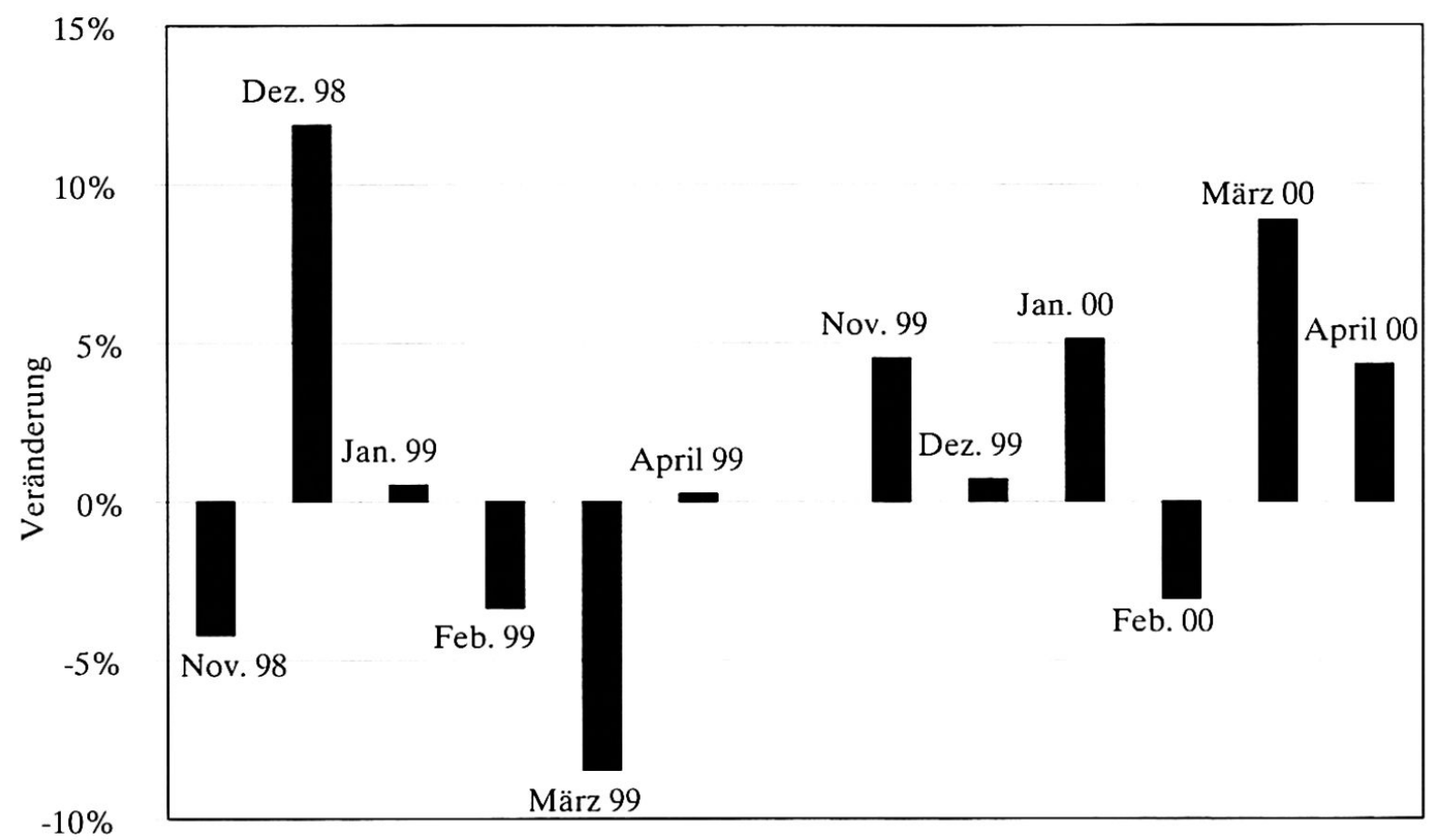

Abb. 8: Entwicklung der Hotellogiernächte in den Schweizer Bergkurorten in den einzelnen Monaten der Winterhalbjahre 1998/99 und 1999/00 verglichen mit den Durchschnittswerten der fünf Winterhalbjahre 1993/94 bis $1997 / 98$.

Development of overnight stays in Swiss mountain resort hotels in the different months of the winter seasons 1998/99 and 1999/00 compared with the average of the winter seasons 1993/94 to 1997/98.

Evolution mensuelle des nuitées dans les hôtels de montagne suisses durant les saisons d'hiver 1998/99 et 1999/00 en comparaison avec la moyenne des saisons d'hiver 1993/94 à 1997/98.

Quelle: BFS (Bundesamt für Statistik) 1993-2001

das Ereignis gebunden ist, können indirekte Auswirkungen auch in der Folgezeit noch auftreten. Für den Lawinenwinter 1999 wurde untersucht, wie sich dieser mittelfristig auf den Rest der Wintersaison und längerfristig auf den Februar 2000 ausgewirkt hat. Es liegen Ergebnisse zu den Hotellogiernächten und den Bergbahnen vor.

Bei den Hotels im schweizerischen Alpenraum zeigt sich das auf den ersten Blick vielleicht erstaunliche Ergebnis, dass der Rückgang der Logiernächte im März 1999 wesentlich grösser war als im Februar (vgl. Abbildung 8). So ergibt sich aus der Statistik für das Berggebiet im Februar 1999 eine Abnahme der Hotellogiernächte um $3.4 \%$ verglichen mit dem Durchschnittswert der fünf Vorjahre (nach BFS 1993-2001). Im März nahmen die Übernachtungen dagegen um $8.5 \%$ ab. Im Februar hatten immerhin $40 \%$ der Fremdenverkehrsorte in den Schweizer Alpen noch eine Logiernächtezunahme ausgewiesen; im März waren es nur noch rund $20 \%$. Der Grund für diese Entwicklung ist wohl darin zu suchen, dass die Mehrheit der Hotelgäste bereits in ihrem Ferienort war, als sich im
Februar 1999 die Lawinengefahr erhöhte. Sie konnten also allenfalls früher abreisen, aber kaum noch den ganzen Aufenthalt abbuchen. Personen, welche für den März ein Zimmer gebucht hatten, hatten die Möglichkeit zum Abbuchen dagegen und machten offenbar auch davon Gebrauch. Der April 1999 war bereits wieder weniger stark vom Lawinenwinter betroffen als der März: Die Hotellogiernächte von Ausländern nahmen zwar im Vergleich zum Schnitt der Vorjahre um $2.4 \%$ ab. Weil aber $4.2 \%$ mehr Übernachtungen von Schweizern erzielt wurden, blieb das Aprilergebnis für die Hotellerie im langjährigen Durchschnitt $(+0.3 \%)$.

Der Anteil der Stammgäste an den Wintersporttouristen im schweizerischen Berggebiet ist im Allgemeinen hoch (vgl. Künzl \& Schmidhauser 1989: 8). Es war deshalb zu vermuten, dass man es vor allem im Februar 2000 bemerken müsste, falls Gäste ausbleiben würden, die durch die Lawinenereignisse im Vorjahr abgeschreckt worden sind. Die Auswertung der Logiernächtezahlen zeigt, dass die Hotelübernachtungen in den Bergkurorten im Februar 
2000 praktisch auf dem Vorjahresniveau und damit nach wie vor $3 \%$ unter dem Durchschnitt der fünf Jahre vor dem Lawinenwinter lagen (vgl.Abbildung 8). Der Februar ist in der Wintersaison 1999/2000 der einzige Monat, in welchem die Zahl der Hotellogiernächte unterdurchschnittlich war. Dies lässt vermuten, dass doch eine gewisse Zahl von Gästen durch die Lawinenereignisse abgeschreckt worden sein muss. Andererseits wäre als Langzeitwirkung auch eine weitere Abnahme der Übernachtungszahlen denkbar gewesen, die aber nicht eingetreten ist.

Ein weiteres Jahr später, im Februar 2001, waren dann keine Auswirkungen des Lawinenwinters 1999 mehr zu spüren: Im Vergleich zum Durchschnitt der fünf Jahre vor 1999 nahmen die Hotellogiernächte in den Bergkurorten um 3.6\% zu, im Vergleich zum Februar 1999 sogar um $7.2 \%$.

Während die Zahl der Hotellogiernächte Auskunft zu den übernachtenden Gästen geben kann, weisen die Bergbahnfrequenzen auch auf das Verhalten der Tagesgäste hin. Es zeigt sich dabei ein etwas anderes Bild: Die Bergbahnen waren nämlich vor allem kurzfristig vom Lawinenwinter 1999 betroffen. Wie bereits erwähnt, hatten in der SLF-Umfrage $88 \%$ der Bergbahn- und Skiliftunternehmen angegeben, sie hätten im Februar 1999 im Vergleich zum Vorjahr Mindereinnahmen gehabt (vgl. Abbildung 9). Für den März und den April 1999 traf dies nur noch auf 45\% der Betriebe zu. $36 \%$ hatten in diesen Monaten durchschnittliche Frequenzen und 19\% sogar überdurchschnittliche. Eine Zunahme der Passagierfahrten wurde damit erklärt, dass bei Wintersportlern Nachholbedarf vorhanden gewesen sei und dass die Schneeverhältnisse ausgezeichnet waren. Langfristig scheinen die Folgen des Lawinenwinters für die Bergbahnen eher gering gewesen zu sein: Nur $11 \%$ der Unternehmen erklärten, sie hätten im Februar 2000 immer noch unterdurchschnittliche Passagierfrequenzen gehabt, weil die Gäste im Vorjahr abgeschreckt worden seien. $49 \%$ der Unternehmen gaben an, sie hätten im Februar 2000 wieder durchschnittliche Fahrgastzahlen erreicht und 3\% bezeichneten die Frequenzen als überdurchschnittlich. Die übrigen machten keine Aussage zur Entwicklung der Passagierzahlen. Praktisch alle erklärten jedoch, der Lawinenwinter 1999 sei bei ihnen ein Jahr danach kein Thema mehr gewesen.

Aus den vorliegenden Angaben lassen sich folgende Schlüsse zu den mittel- und längerfristigen Folgen von katastrophalen Naturereignissen auf die Tourismusbranche in den Alpen ziehen: Auf den Tagestourismus und die davon abhängigen Branchen wirkt sich ein Naturereignis sofort aus. Mittelfristig, d.h. in den Folgemonaten, erholt sich der Tagestourismus aber vermutlich ziemlich rasch. Ein Jahr nach dem Ereignis ist er kaum noch beeinträchtigt. Auch für das Beher-
Februar 1999

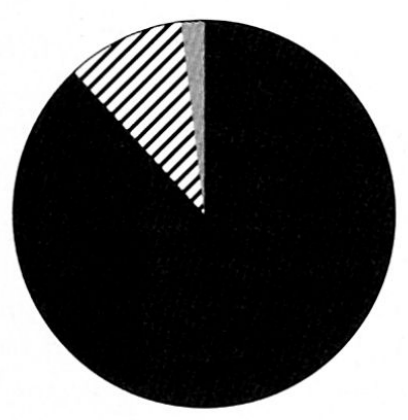

Abnahme
März/April 1999

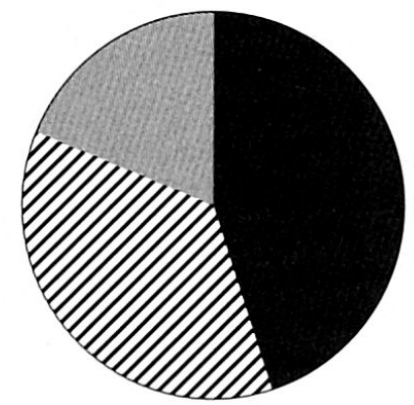

Keine Änderung
Februar 2000

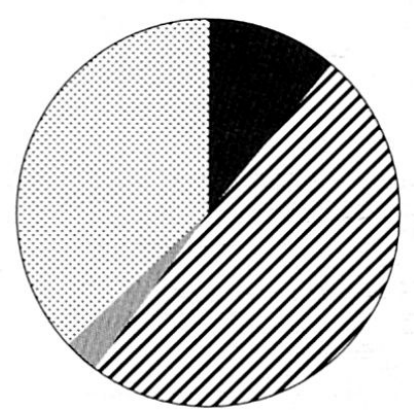

Zunahme

Der Lawinenwinter war ein Jahr danach kein Thema mehr

Abb. 9: Kurz-, mittel- und längerfristige Auswirkungen des Lawinenwinters 1999 auf die Passagierzahlen bzw. Einnahmen der Schweizer Bergbahn- und Skiliftunternehmen. Angaben in \% der Betriebe.

Short term, middle term and long term effects of the avalanche winter of 1999 on the number of passengers resp. the earnings of the Swiss cable car and ski lift companies (in percentages of the total number of companies)

Effets des avalanches de l'hiver 1999 sur le nombre des passagers ainsi que sur le chiffre d'affaires des sociétés de remontées mécaniques suisses à court, moyen et long terme (en pourcentage de toutes les entreprises)

Quelle: NöTHIGER, BrüNDL \& Ammann 2001: 29 
bergungsgewerbe kommt es unmittelbar während eines Ereignisses zu Ausfällen, z.B. wegen gesperrten Zufahrtswegen oder Evakuierungen. Der anteilmässig grösste Rückgang von Übernachtungen dürfte aufgrund von Abbuchungen erst mit Verzögerung eintreten. Ein Jahr nach dem Ereignis muss damit gerechnet werden, dass ein Teil der Feriengäste, die dieses Ereignis selber miterlebt haben, ausbleiben wird. Auf noch längere Sicht hinaus dürfte aber auch das Beherbergungsgewerbe nicht mehr von negativen Folgen eines katastrophalen Naturereignisses betroffen sein.

\subsection{Wie können indirekte Kosten abgeschätzt werden?}

Eine Abschätzung der indirekten Kosten des Lawinenwinters für die Tourismusbranche musste zwangsläufig auf der Grundlage der wenigen Daten geschehen, die nach dem Ereignis erhältlich waren, d.h. vor allem auf Basis der Entwicklung der Hotellogiernächte. Um daraus finanzielle Folgen abschätzen zu können, wurden touristische Wertschöpfungsstudien zu Hilfe genommen.

Diese Studien haben zum Ziel, die finanzielle Bedeu- tung des Tourismusgeschäftes für die Wirtschaft einer bestimmten Region zu eruieren. Dazu sind umfangreiche Befragungen von Touristen und Betrieben erforderlich. Dafür können solche Studien auch Daten zu Touristen liefern, die sonst nie erfasst werden, z.B. zu Tagestouristen und Gästen, die im eigenen Ferienhaus übernachten. Der Nutzen von Wertschöpfungsstudien für die Abschätzung von Mindereinnahmen liegt in erster Linie darin, dass dafür immer auch die Tagesausgaben verschiedener Gästegruppen erhoben werden. Ist nämlich bekannt, wie viel ein durchschnittlicher Gast pro Tag ausgibt, kann abgeschätzt werden, zu welchen Mindereinnahmen das Ausleiben einer bestimmten Anzahl Gäste führt.

Für die Abschätzung der Folgen des Lawinenwinters wurden die Wertschöpfungsstudien der Regionen «Mittelbünden» (ZegG, RüTter \& Riner 1993), «Berner Oberland» (RÜTTER et al. 1995) und «Wallis» (RütTER et al. 2001) benutzt. Es handelt sich dabei um die umfangreichsten derartigen Studien, die in der Schweiz bis heute durchgeführt wurden. Verwendet wurden die durchschnittlichen Tagesausgaben der Wintergäste nach Gästegruppen und Ausgabenkate-

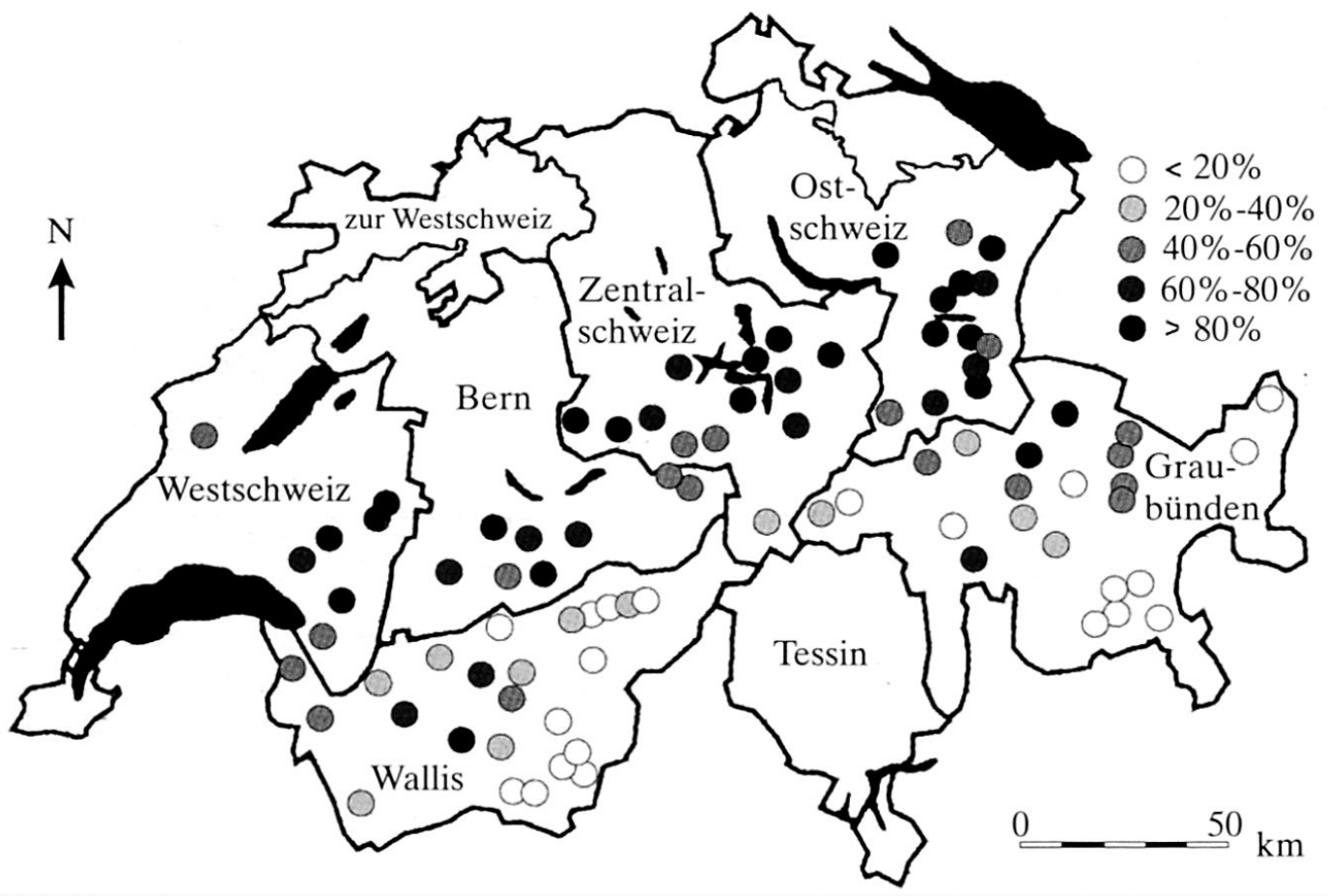

Abb. 10: Geschätzter Anteil der Tagesgäste an der Gesamtzahl aller Fahrgäste bei den Bergbahn- und Skiliftunternehmen, die sich an der Umfrage des SLF beteiligten.

Estimated percentage of day-trippers in relation to the total number of passengers of the Swiss cable car and ski lift companies who took part in the SLF survey.

Dépendance des entreprises de remontées mécaniques par rapport au tourisme journalier: pourcentage estimé des touristes journaliers par rapport aux usagers totaux des entreprises qui ont participé à l'enquête de l'ENA.

Quelle: NÖTHIGER, BrüNDL \& AmMANN 2001: 27 
gorien sowie das zahlenmässige Verhältnis der verschiedenen Gruppen von Übernachtungsgästen zueinander. Der durchschnittliche Anteil der Tagestouristen an der Gesamtzahl der Gäste variiert in den einzelnen Studien recht stark. Die SLF-Umfrage bei den Bergbahn- und Skiliftunternehmen zeigte die Ursache dieser Differenzen: In Wintersportgebieten der Voralpen beträgt der Anteil der Tagesgäste häufig über $80 \%$, in den inneren Alpen dagegen z.T. unter $20 \%$ (vgl. Abbildung 10). Für die Schätzung wurde ein mittlerer Wert von 50\% angenommen.

Bei den Übernachtungsgästen wurde davon ausgegangen, dass deren Zahl in allen Unterkunftskategorien in anteilmässig gleichem Umfang wie in der Hotellerie zurückgegangen ist. Zusätzlich wurde angenommen, dass die Tagestouristen im Februar 1999 während durchschnittlich 15 Tagen ausgefallen sind. Diese Annahme dürfte realistisch sein, wenn man berücksichtigt, dass in diesem Monat während etwa 17 Tagen intensive Niederschläge fielen und teilweise sehr grosse Lawinengefahr herrschte. Für März und April 1999 sowie Februar 2000 wurde aufgrund der Erkenntnisse über die mittel- und längerfristigen Folgen des Lawinenwinters (vgl. Abschnitt 5.4) kein Ausfall von Tagesgästen angenommen.

Es ergeben sich in der durchgeführten Schätzung totale Mindereinnahmen von 328 Mio. SFr. für die Tourismusbranche im Schweizer Alpenraum (vgl. Tabelle
3), wobei $93 \%$ des Gesamtbetrages auf das Jahr 1999 entfallen. Dies ist eine beträchtliche Summe, auch im Vergleich zu den 439 Mio. Fr. an bekannten direkten Schäden des Lawinenwinters 1999. Sie macht die grosse Bedeutung der bisher eher vernachlässigten indirekten Schäden von Naturereignissen nochmals eindrücklich klar.

\subsection{Wie könnten indirekte Kosten in Zukunft gesenkt werden?}

Die beste Methode, um indirekte Kosten eines katastrophalen Naturereignisses zu senken, ist natürlich, das Eintreten des Ereignisses selber zu verhindern. Gerade im schweizerischen Alpenraum wurden die Einrichtungen zum Siedlungs- und Verkehrsachsenschutz in den letzten 50 Jahren sehr stark ausgebaut und haben sich während des Lawinenwinters auch bewährt. Ein weiterer Ausbau ist zwar punktuell möglich, der bauliche Lawinenschutz wird aber auch in Zukunft bei derartigen Jahrhundertereignissen an seine Grenzen stossen. Es müssen deshalb weitere Massnahmen zur Reduktion indirekter Kosten ins Auge gefasst werden.

Um herauszufinden, welche Massnahmen von den Betroffenen nach dem Lawinenwinter 1999 ergriffen wurden, wurden die Bergbahn- und Skiliftunternehmen auch dazu befragt (NÖTHIGER, BRÜNDL \& AMMANN 2001: 30f.). Zwei Dritteln der befragten Betriebe erschien es überhaupt nicht nötig, etwas Spezielles zu

\begin{tabular}{lcccccc}
\hline & $\begin{array}{c}\text { Beherber- } \\
\text { gung }\end{array}$ & $\begin{array}{c}\text { Gast- } \\
\text { stätten }\end{array}$ & $\begin{array}{c}\text { Detail- } \\
\text { handel }\end{array}$ & $\begin{array}{c}\text { Berg- } \\
\text { bahnen }\end{array}$ & Übriges & $\begin{array}{c}\text { Alle } \\
\text { Branchen }\end{array}$ \\
\hline Feb. 1999 & -10.0 Mio. & -102.8 Mio. & -36.4 Mio. & -79.9 Mio. & -17.8 Mio. & $\mathbf{- 2 4 6 . 9 ~ M i o . ~}$ \\
März 1999 & -22.1 Mio. & -13.5 Mio. & -9.6 Mio. & -8.0 Mio. & -4.7 Mio. & $\mathbf{- 5 8 . 0 ~ M i o . ~}$ \\
April 1999 & +0.3 Mio. & +0.2 Mio. & +0.1 Mio. & +0.1 Mio. & +0.1 Mio. & $+\mathbf{+ 0 . 7}$ Mio. \\
\hline Total 1999 & -31.9 Mio. & -116.2 Mio. & -45.9 Mio. & -87.7 Mio. & -22.4 Mio. & $\mathbf{- 3 0 4 . 2 ~ M i o . ~}$ \\
Feb. 2000 & -9.1 Mio. & -5.6 Mio. & -4.0 Mio. & -3.3 Mio. & -1.9 Mio. & $\mathbf{- 2 3 . 9 ~ M i o . ~}$ \\
\hline Total & $\mathbf{- 4 1 . 0 ~ M i o . ~}$ & $\mathbf{- 1 2 1 . 7 ~ M i o . ~}$ & $\mathbf{- 4 9 . 9 ~ M i o . ~}$ & $\mathbf{- 9 1 . 0 ~ M i o . ~}$ & $\mathbf{- 2 4 . 3 ~ M i o . ~}$ & $\mathbf{- 3 2 8 . 1}$ Mio. \\
\hline
\end{tabular}

Tab. 3: Vermutliche indirekte finanzielle Auswirkungen des Lawinenwinters 1999 auf die Tourismusbranche im schweizerischen Alpenraum (in CHF). Zu «Übriges» gehören z.B. Schneesportschulen, lokale Busbetriebe, Heilbäder, Museen, Kinos und Kongressveranstalter.

Probable indirect loss of earnings caused by the avalanche winter of 1999 for the tourist industry in the Swiss Alps (in $C H F$ ).

Les pertes de chiffre d'affaires supposées pour la branche touristique de la zone de montagne suisse causées par suite des avalanches de l'hiver 1999 (en CHF).

Quelle: eigene Berechnungen auf der Basis von Daten des BFS (1993-2001) sowie von Wertschöpfungsstudien 
unternehmen, die anderen ergriffen eine oder mehrere Massnahmen (vgl. Abbildung 11 oben). Mit 13\% die grösste Gruppe versuchte über aktive Medienarbeit ein positives Bild ihres Wintersportgebietes zu vermitteln. Bezüglich der Beurteilung des Erfolges der getroffenen Massnahmen lässt sich kein Trend ausmachen: Sie fiel allgemein sehr unterschiedlich aus.

Die Bergbahnunternehmen wurden auch gefragt, welche Massnahmen gegen die indirekten Auswirkungen zukünftiger katastrophaler Naturereignisse ergriffen werden sollten. Hier fand nur ein Viertel der Betriebe, die heutigen Massnahmen reichten aus. Die übrigen sahen weiteren Handlungsbedarf (vgl. Abbildung 11 unten). Lediglich $10 \%$ sprachen sich dabei für zusätzliche technische Schutzmassnahmen aus, was ebenfalls auf deren hohen Ausbaustandard in der Schweiz hinweist. Etwa ein Viertel der Unternehmen würde die Einrichtung einer Versicherung oder eines
Fonds begrüssen, welcher Tourismusbetriebe nach katastrophalen Naturereignissen auch dann für Mindereinnahmen entschädigt, wenn herkömmliche Versicherungen nicht greifen. Hinsichtlich der wirtschaftlichen und technischen Machbarkeit wäre diese Idee sicher prüfenswert.

$29 \%$ der Betriebe sehen in der verbesserten Kundeninformation noch Potential für die Zukunft. In Krisensituationen sollen die Gäste z.B. über Lautsprecher, Internet, Lokalradio oder Anzeigetafeln schneller und präziser über die Lage aufgeklärt werden. Ebenfalls mit der Informationspolitik hängt die stärkere Einflussnahme auf die Medienberichterstattung zusammen, welche sogar von 39\% der Bergbahn- und Skiliftunternehmen als wichtige Massnahme für die Zukunft betrachtet wird. Konkret wurde eine Professionalisierung der Medienbetreuung im Krisenfall gefordert. Informationspolitik ist aber nicht nur den Bergbahnen

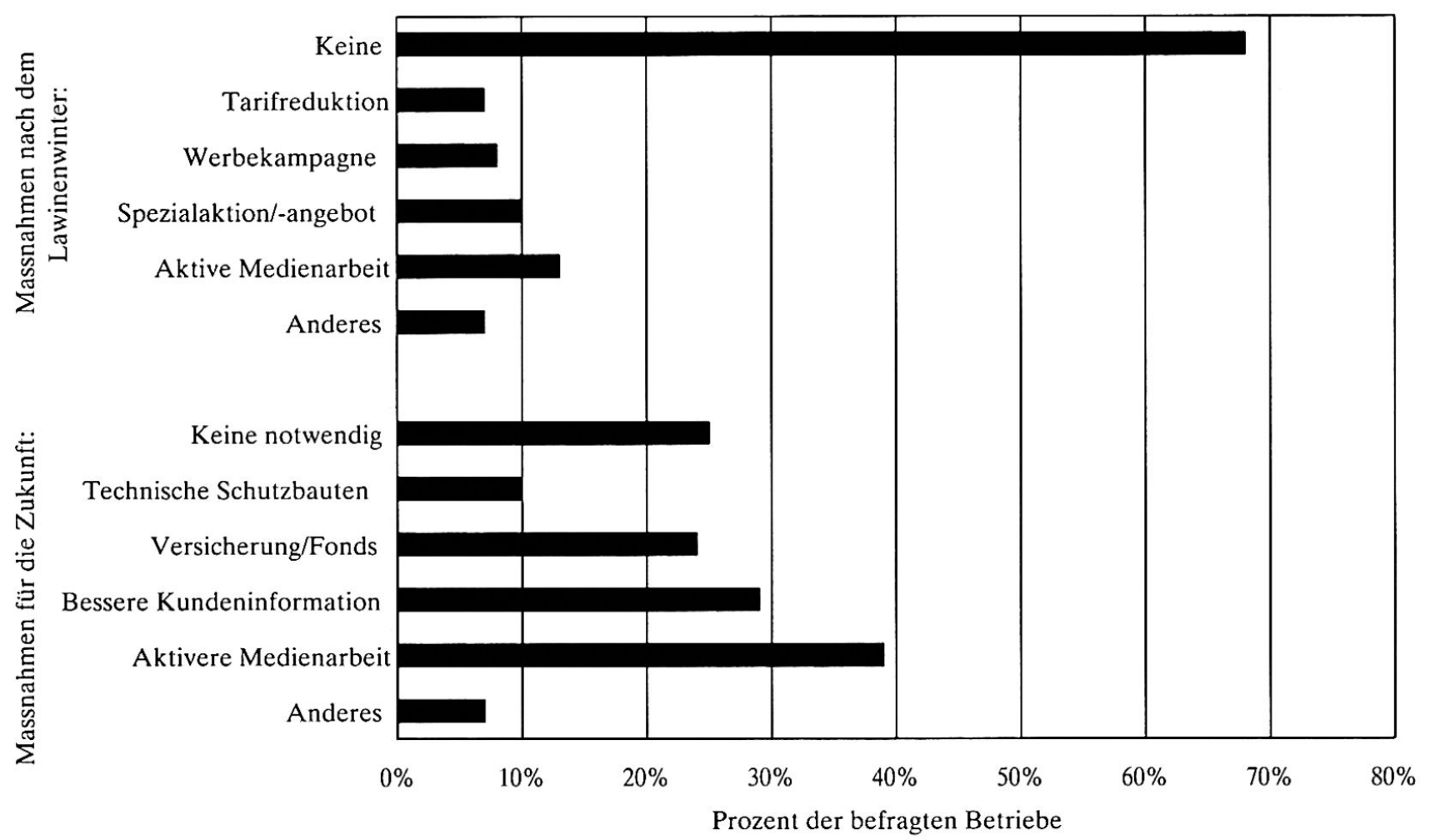

Abb. 11: Besondere Massnahmen, welche von den Schweizer Bergbahn- und Skiliftunternehmen nach dem Lawinenwinter 1999 ergriffen wurden bzw. von diesen für die Zukunft vorgeschlagen werden, um indirekte Kosten von Naturereignissen zu senken (in \% aller Betriebe). Mehrere Antworten waren möglich.

Special measures which have been taken by the Swiss cable car and ski lift companies after the avalanche winter of 1999 and which they have suggested for the future in order to decrease the indirect costs of such events (in percentages of the total number of companies). More than one measure was possible.

Mesures spéciales prises après les avalanches de l'hiver 1999 par les sociétés de remontées mécaniques suisses et propositions de celles-ci pour l'avenir, afin de diminuer les coûts indirects de pareils événements (en pourcentage de toutes les entreprises). Plusieurs réponses étaient possibles.

Quelle: NöTHIGER, BRÜNDL \& AMmANn 2001: 30f. 
wichtig: Auch in der Umfrage bei den Tourismusbetrieben in Davos schlug ein Drittel der Befragten spontan Verbesserungen im Bereich der Information vor (NöTHiger \& Ammann 2001: 9). Gefordert wurde z.B. die Einrichtung einer zentralen Medien- und Informationsstelle für die gesamte Gemeinde, über die alle Informationen zur Krisensituation sowohl für die Gäste als auch für die Presse laufen sollten.

Dazu gilt es allerdings zu bedenken, dass $8 \%$ der befragten Davoser Feriengäste spontan bemerkten, die Informationen müssten in einer solchen Situation «vertrauenswürdig» und «neutral» sein (NöTHIGER \& AMMANN 2001: 14). Als vertrauenswürdig wurden namentlich die Polizei und die Behörden bezeichnet. Bei anderen Quellen wurde der Verdacht geäussert, sie würden aufgrund wirtschaftlicher Interessen nicht objektiv informieren. Erwähnt wurden in diesem Zusammenhang der Verkehrsverein, die Bergbahnen und das Fernsehen. Diese Vorbehalte müssten bei der Informationspolitik nach zukünftigen Naturereignissen berücksichtigt werden. Falls tatsächlich vermehrt professionelle Informationsstellen für Krisensituationen eingerichtet würden, wäre für diese Aufgabe eine öffentliche Institution einem Privatunternehmen vorzuziehen, um den Verdacht von Befangenheit von vornherein zu vermeiden. Als Beispiel einer Informationsquelle, welche diese Anforderungen in Zukunft einmal erfüllen könnte, sei das Internet-Projekt für ein «Interkantonales Frühwarn- und Kriseninformationssystem IFKIS» genannt. Dessen Konzept wird gegenwärtig am SLF erarbeitet. Im Rahmen dieses Projekts wird auch eine Plattform geschaffen, mit der breite Bevölkerungskreise und die Medien in einer Region über Sicherungsmassnahmen informiert werden können.

\section{Schlussfolgerungen}

Die wichtigsten Erkenntnisse zu den indirekten Auswirkungen von Naturereignissen, die sich aus der Untersuchung der Folgen des Lawinenwinters 1999 für die Tourismusbranche in den Schweizer Alpen ergaben, sind:

- Für die Tourismusbranche führte der Lawinenwinter in erster Linie zu indirekten Kosten, d.h. zu Mindereinnahmen, währenddem direkte Lawinen- oder Schneedruckschäden - z.B. an Gebäuden oder Bergbahnen - eine eher geringe Rolle spielten.

- Neben der Sperrung von Zufahrtswegen sind wahrscheinlich vor allem die Ausserbetriebnahme von Bergbahnen, das schlechte Wetter während der intensiven Schneefälle im Februar 1999 und die Medienberichte über die Lawinensituation für die Mindereinnahmen im Tourismussektor verantwortlich.
- Während die direkten Schadenkosten des Lawinenwinters 1999 von der öffentlichen Hand und von Versicherungen weitgehend abgedeckt waren, fielen die indirekten Kosten fast vollständig zu Lasten der Betroffenen.

- Positive wirtschaftliche Auswirkungen des Lawinenwinters für den Tourismus, wie die Umlagerung von Gästen auf lawinensichere Gebiete oder Zusatzeinnahmen durch «Sensationstouristen» haben sich in Grenzen gehalten.

- Währenddem der Tagestourismus hauptsächlich im Februar 1999 durch die Lawinenereignisse beeinträchtigt wurde, wirkten sich diese auf den Beherbergungssektor mit einer gewissen Verzögerung aus und zeitigten sogar im Februar 2000 noch gewisse Langzeitfolgen.

- Gemäss aktueller Schätzung betrugen die indirekten Kosten des Lawinenwinters für die Tourismusbranche im schweizerischen Alpenraum 328 Mio. SFr. Diese Summe ist auch im Vergleich zu den direkten Schadenkosten von 439 Mio. SFr. beträchtlich und macht die Bedeutung der indirekten Kosten deutlich.

- Als Massnahme, um die indirekten Auswirkungen von zukünftigen Naturereignissen möglichst tief zu halten, steht für die Tourismusbranche im Schweizer Alpenraum eine Verbesserung der Informationsund Medienpolitik im Vordergrund.

\section{Literatur}

BFS (Bundesamt für Statistik) (1993-2001): Hotel- und Kurbetriebe in der Schweiz. Angebot und Nachfrage. November 1993 - April 2001 (Monatsübersichten). Neuchâtel: BFS.

IKAR (Internationale Kommission für Alpines Rettungswesen) (1999): Protokoll der 51. Delegiertenversammlung der IKAR vom 25. September 1999 in Sonthofen, Oberallgäu (Deutschland). Avalanche sub-committee report. - http://www1.vrz.net/public/ ikar-cisa.nsf/ProtokolleDeutsch +Lawinen/ 16FACDC297F14742C125683800698133 25.09.1999.

KrisenSTAB Brig (Hrsg.) (1994): Unwetterkatastrophe Brig-Glis 24. September 1993. Die Katastrophenbewältigung: Erfahrungen und Erkenntnisse des Krisenstabes. - Brig: Krisenstab Unwetterkatastrophe Brig-Glis 1993.

KüNZI, G.F. \& H.P. Schmidhauser (1989): TOMAS - Touristisches Marktforschungssystem. Expertenbericht Winterhalbjahr 1988/89, Bern: Schweizer Tourismus-Verband - STV.

Nöthiger, Ch.J. \& W.J. Ammann (2001): Die Auswirkungen des Lawinenwinters 1999 auf die Betriebe und die Feriengäste in der Gemeinde Davos (Kanton Graubünden). - = Eidg. Institut für Schnee- und Lawinenforschung SLF, Interner Bericht Nr. 744, Davos: $1-24$. 
NöTHIGER, Ch.J. (2000): Der Lawinenwinter 1999. Fallstudie Elm (Kanton Glarus). Indirekte Auswirkungen auf die lokale Wirtschaft. - Davos: Eidg. Institut für Schnee- und Lawinenforschung SLF.

Nöthiger, Ch.J., Bründl, M. \& W.J. Ammann (2001): Die Auswirkungen der Naturereignisse 1999 auf die Bergbahn- und Skiliftunternehmen der Schweiz. - In: Tourist Review/Zeitschrift für Fremdenverkehr/Revue de Tourisme 01/1:23-32.

RÜTreR, H. et al. (1995): Tourismus im Kanton Bern. Wertschöpfungsstudie. $-=$ Berner Studien zu Freizeit und Tourismus 34, Rüschlikon/Bern: 1-261.

RüTteR, H. et al. (2001): Der Tourismus im Wallis. Wertschöpfungsstudie. - Sitten: Dienststelle für Tourismus- und Wirtschaftsförderung.

SChNeEbeli, M., Laternser, M. \& W. Ammann (1997): Destructive snow avalanches and climate change in the Swiss Alps. - In: Eclogae Geologicae Helvetiae. Zeitschrift der schweizerischen geologischen Gesellschaft 90/3: 457-61.

SLF (Eidg. Institut für Schnee- und Lawinenforschung) (2000): Der Lawinenwinter 1999. Ereignisanalyse. Davos: Eidg. Institut für Schnee- und Lawinenforschung SLF.

STENGER, M. (1998): Repräsentativerhebungen im Tourismus. Ein methodischer und inhaltlicher Vergleich. - = Materialien zur Fremdenverkehrsgeographie, Heft 45, Trier: 1-145.

ZEGG, R., RüTter, H. \& A. Riner (1993): Die wirtschaftliche Bedeutung des Tourismus in der Region Mittelbünden. Gesamtbericht mit Schlussresultaten und Methodik. - Chur: Grischconsulta AG.

\section{Zusammenfassung: Indirekte Auswirkungen von Naturgefahren auf den Tourismus - Das Beispiel des Lawinenwinters 1999 in der Schweiz}

Der vorliegende Artikel befasst sich mit den sogenannten «indirekten» Auswirkungen von katastrophalen Naturereignissen, worunter vor allem Mindereinnahmen für die Wirtschaft verstanden werden. Präsentiert werden Untersuchungsergebnisse zu den indirekten Kosten des Lawinenwinters 1999 für die Tourismusbranche in den Schweizer Alpen. Ursachen für diese Kosten waren v.a. Strassensperrungen, ausser Betrieb genommene Bergbahnen, das schlechte Wetter und wahrscheinlich auch Medienberichte. Tagestouristen blieben in erster Linie im Februar 1999 aus, übernachtende Gäste zusätzlich auch im März 1999 und teilweise sogar noch im Februar 2000. Insgesamt führte der Lawinenwinter 1999 für die Tourismusbetriebe im Schweizer Berggebiet zu Mindereinnahmen von schätzungsweise rund 330 Mio. Franken. Diese indirekten Kosten mussten zum überwiegenden Teil von den betroffenen Betrieben selbst getragen werden. Die Umlagerung eines Teils der Touristenströme auf lawinensichere Gebiete und das gelegentliche Auftre- ten von «Sensationstouristen» nach den Ereignissen konnte die Mindereinnahmen nicht kompensieren. Als Massnahmen, um in Zukunft die indirekten Kosten von derartigen Naturereignissen möglichst tief halten zu können, stehen Verbesserungen in der Informations- und Medienpolitik im Vordergrund.

\section{Summary: Indirect Effects of Natural Hazards on Tourism - Exemplified by the avalanche winter of 1999 in Switzerland}

This article deals with the so-called «indirect» effects of natural hazards, loss of earnings being the main effect. The results presented here derive from a survey carried out on the indirect costs of the avalanche winter of 1999 for the tourist industry in the Swiss Alps. These costs are mostly the result of the closure of access roads, the decommissioning of several cable cars and ski lifts, the bad weather conditions and probably press coverage as well. Day-trippers primarily stayed away during February 1999, overnight guests also in March 1999 and to a degree even in February 2000. The total loss of earnings for the tourist industry in the Swiss Alps caused by the avalanche winter of 1999 adds up to approximately 330 Mio. Swiss Francs, costs which were almost entirely carried by the companies affected. The transfer of tourists to winter sport areas not affected by the avalanches or the occasional occurrence of «sensationalist tourists» after the events could not compensate for these losses. Improved public relations is considered to be the most important measure to cope with the indirect costs of natural hazards in the future.

\section{Résumé: Effets indirects des dangers naturels sur le tourisme - L'exemple des avalanches de l'hiver 1999 en Suisse}

Le présent article traite des effets dits «indirects» des dangers naturels qui s'expriment principalement en terme de pertes de chiffre d'affaires pour l'économie. Les résultats présentés proviennent de recherches effectuées sur les coûts indirects des avalanches de l'hiver 1999 pour la branche touristique des Alpes suisses. Les causes principales de ces coûts étaient: le blocage des routes d'accès, le non-fonctionnement des installations de remontées mécaniques, le mauvais temps et probablement aussi les reportages des médias. Les touristes journaliers sont principalement restés chez eux en février 1999, les hôtes de vacances sont également restés chez eux en mars 1999 et partiellement aussi en février 2000. Au total, les pertes de chiffre d'affaires pour la branche touristique de la zone de montagne suisse s'élèvent vraisemblablement à environ 330 millions de francs suisses, presque entièrement au dépens des compagnies affectées. Le transfert d'un certain nombre de touristes vers des stations touristiques non-affectées par des avalanches ou la présence occasionnelle de «touristes de badau- 
dage» après les évènements de février 1999 pourraient à peine compenser ces pertes. Améliorer les relations publiques peut être considéré comme la mesure la plus importante pour diminuer les coûts indirects de tels évènements à l'avenir.

Dipl. Geogr. Christian J. Nöthiger, Dr. Michael Bründl, Dr. Walter J. Ammann, Eidgenössisches Institut für Schnee- und Lawinenforschung SLF, Flüelastrasse 11, CH-7260 Davos.

Prof. Dr. Hans Elsasser, Geographisches Institut der Universität Zürich, Winterthurerstrasse 190, CH-8057

Zürich.

e-mail:

noethiger@slf.ch

bruendl@slf.ch

ammann@slf.ch

elsasser@geo.unizh.ch

Manuskripteingang/received/manuscrit entré le

2.1.2002

Annahme zum Druck/accepted for publication/accepté pour l'impression: 23.5 .2002 